\title{
Transformation of Hyperfine Interactions in Co-Substituted Nanoperm-Type Alloys during Annealing
}

\author{
T. HAtala ${ }^{a, *}$ And M. MiglieRini ${ }^{a, b}$ \\ ${ }^{a}$ Slovak University of Technology, Ilkovičova 3, 81219 Bratislava, Slovakia \\ ${ }^{b}$ Centre for Nanomaterial Research, Palacky University, Šlechtitelů 11, 77146 Olomouc, Czech Republic \\ Structure and magnetic order of rapidly quenched $\left(\mathrm{Fe}_{1-x} \mathrm{Co}_{x}\right)_{79} \mathrm{Mo}_{8} \mathrm{Cu}_{1} \mathrm{~B}_{12}$ alloys is investigated by ${ }^{57} \mathrm{Fe}$ \\ Mössbauer spectrometry in transmission geometry and by conversion electron Mössbauer spectrometry. The effect \\ of heat treatment upon hyperfine interactions was studied as a function of Co contents $(x=0,0.5)$. Magnetic \\ dipole interactions developed in both alloys when sufficient number of ferromagnetic nanograins is created after \\ heat treatment. The kinetics of crystallization was derived from in situ diffraction of synchrotron radiation. The \\ progress of crystallization is different at both sides of the ribbon-shaped samples.
}

PACS numbers: 76.80.+y, 71.55.Jv, 42.25.Fx, 75.75.-c

\section{Introduction}

Nanocrystalline metallic alloys represent a new class of materials the physical properties of which can be tailored not only by the help of their chemical composition but also by varying the size and amount of crystalline grains [1]. Thus, structural transformations belong to principal mechanisms that considerably affect also their magnetic properties.

$\left(\mathrm{Fe}_{1-x} \mathrm{Co}_{x}\right)_{79} \mathrm{Mo}_{8} \mathrm{Cu}_{1} \mathrm{~B}_{12}$ alloys for $x=0$ and 0.5 were prepared by the method of planar-flow casting. Magnetic order of the alloys both in the as-quenched (amorphous) state and after heat treatment (nanocrystalline state) was followed by ${ }^{57} \mathrm{Fe}$ Mössbauer spectrometry in transmission geometry (TMS) and by conversion electron Mössbauer spectrometry (CEMS) using a ${ }^{57} \mathrm{Co}(\mathrm{Rh})$ source mounted on a standard constant acceleration spectrometer working at room temperature. The Mössbauer effect experiments were performed at room temperature upon samples that were annealed at selected temperatures for $30 \mathrm{~min}$. Diffraction of synchrotron radiation (DSR) with energy of $7 \mathrm{keV}$, which is available at BESSY KMC-2 station (Berlin), was used for in situ experiments during continuous temperature increase $(10 \mathrm{~K} / \mathrm{min})$. Diffractograms that extend over the principal (110) reflection of a bcc-Fe lattice were collected every $10 \mathrm{~s}$ using a $2 \mathrm{D}$ detector.

\section{Results and discussion}

TMS spectra of as-quenched and annealed $\left(\mathrm{Fe}_{1-x} \mathrm{Co}_{x}\right)_{79} \mathrm{Mo}_{8} \mathrm{Cu}_{1} \mathrm{~B}_{12}$ alloys are plotted in Fig. 1.

\footnotetext{
* corresponding author; e-mail: tomas.hatala@stuba.sk
}

While the Co-free alloy is paramagnetic as evidenced by a doublet-like spectrum in Fig. 1a, addition of Co $(x=0.5)$ significantly alternates the magnetic arrangement. The six-line spectrum in Fig. 1b is characteristic for magnetically ordered amorphous system with an average hyperfine magnetic field of $22.8 \mathrm{~T}$.
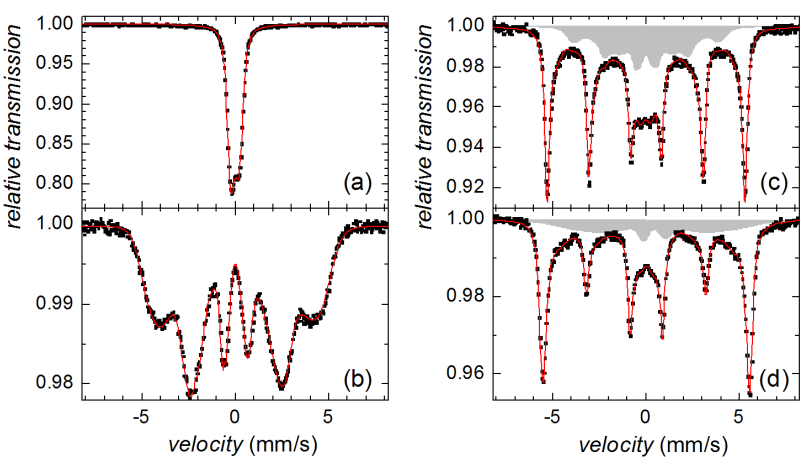

Fig. 1. TMS of $\left(\mathrm{Fe}_{1-x} \mathrm{Co}_{x}\right)_{79} \mathrm{Mo}_{8} \mathrm{Cu}_{1} \mathrm{~B}_{12}$ alloy for $x=0$ (a), (c), and $x=0.5(\mathrm{~b}),(\mathrm{d})$ in as-quenched (a), (b) and nanocrystalline, annealed at $550{ }^{\circ} \mathrm{C}$ (c), (d) states. Grey spectral components represent the residual amorphous phase.

After annealing, bcc-Fe and bcc-Fe,Co nanocrystals have emerged in the $x=0$ and $x=0.5$ alloys, respectively. They are identified from TMS spectra in Fig. 1c and $d$ by narrow sextuplet lines. Magnetic part of the residual amorphous matrix is visualized by grey spectral components which exhibit broad sextuplet lines. They are indication of formation of dipole magnetic hyperfine interactions. The development of their magnetic fields with temperature of annealing is plotted in Fig. 2. The 
appearance and consequent increase in magnetic order for $x=0$ can be associated with changes in composition of the amorphous matrix during progressing crystallization as well as exchange magnetic interactions among nanocrystalline grains which magnetically polarize the originally paramagnetic amorphous matrix [2]. On the other hand, rather strong magnetic interactions observed in as-quenched $x=0.5$ moderately decrease after annealing mainly due to changes in composition of the amorphous rest [3].

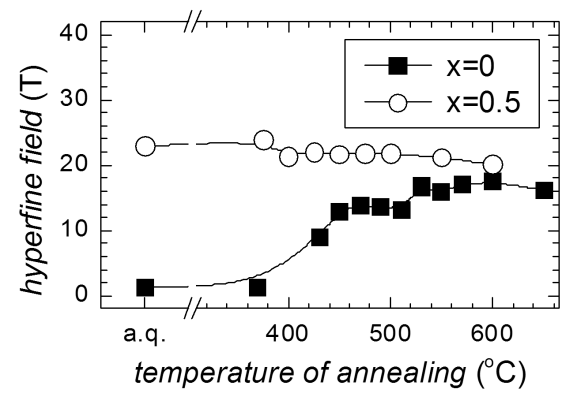

Fig. 2. Average magnetic field plotted against temperature of annealing for the amorphous phase in $\left(\mathrm{Fe}_{1-x} \mathrm{Co}_{x}\right)_{79} \mathrm{Mo}_{8} \mathrm{Cu}_{1} \mathrm{~B}_{12}$ alloys.

Presence of nanocrystallites in as-quenched $x=0$ alloy was checked by DSR and CEMS. DSR pattern in Fig. 3a, which was taken from the air side of the ribbon, demonstrates minor reflection from extremely small $(\approx 2 \mathrm{~nm})$ crystals. They are under the detection limit of CEMS. Consequently, no signal from such small nanocrystals is seen in Fig. 3c. On the wheel side, nanocrystals are detected by both DSR and CEMS. The corresponding Mössbauer lines are marked by arrows in Fig. 3d.
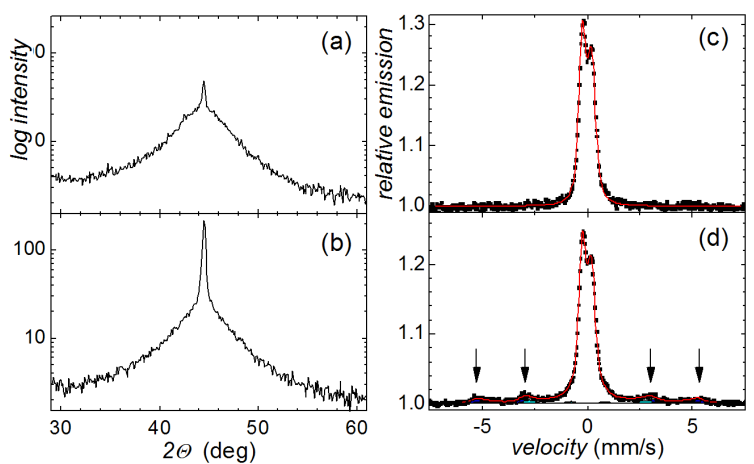

Fig. 3. DSR (a), (b) and CEMS (b), (d) patterns taken from the air (a), (c), and wheel (b), (d) sides of the $x=0$ ribbon. Arrows in (d) indicate spectral lines of bcc-Fe phase.
Progress of crystallization on the wheel side of the $x=0$ ribbon was followed by in situ DSR in Fig. 4a [4]. The temperatures of the onset of the first and the second crystallization were determined to be $403^{\circ} \mathrm{C}$ and $604^{\circ} \mathrm{C}$, respectively. Relative content of nanocrystals and their lattice constants, $a$, derived from the diffractograms are plotted against temperature in Fig. 4b. After the onset of crystallization, the decrease in $a$ indicates that Fe atoms attempt to acquire optimal structural arrangement. However, Mo inclusions in the bcc lattice are responsible for higher $a$-values than in pure $\mathrm{Fe}$ phase. Subsequent tendency of $a$ to increase is associated with lattice expansion due to high temperature.
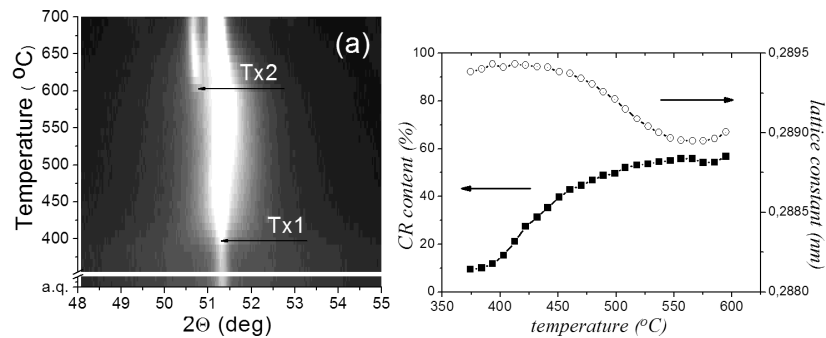

Fig. 4. Contour plot of DSR patterns (a) from the wheel side of the $x=0$ ribbon with indicated crystallization temperature $T_{x 1}=403^{\circ} \mathrm{C}$ and $T_{x 2}=604{ }^{\circ} \mathrm{C}$. Content of crystalline phase and corresponding lattice constants plotted against temperature (b).

\section{Conclusions}

To conclude, substitution of Fe atoms by Co significantly alters the magnetic order. During heat treatment, bcc-Fe and bcc-Fe, Co nanocrystallites develop. Their rising relative content affects also magnetic hyperfine fields of the residual amorphous matrix.

\section{Acknowledgments}

This work was supported by the grants VEGA 1/0033/10, SK-PL-0013-09, and MSM6198959218.

\section{References}

[1] G. Herzer, Phys. Scr. T 49, 307 (1993).

[2] J.S. Garitaonandia, P. Gorria, L. Fernandez Barquin, J.M. Barandiaran, Phys. Rev. B 61, 6150 (2000).

[3] M. Miglierini, J.M. Greneche, J. Phys., Condens. Matter 15, 5637 (2003).

[4] M. Miglierini, J. Non-Cryst. Solids 354, 5093 (2008). 